\title{
Cardioprotective Potential of Baicalein: A Short Review of In Vitro and In Vivo Studies
}

\section{Chen Bao-An ${ }^{1,2 *}$, Ravichandran Senthilkumar ${ }^{1,2}$, Fu Rong ${ }^{3}$ and Qing long Guo ${ }^{3}$}

${ }^{1}$ Department of Neoplastic Hematologic Disorders (Medical Science Key Subject of Jiangsu Province), Zhongda Hospital, Schoool of Medicine, Southeast University, Nanjing 210009, P.R. China

${ }^{2}$ Department of Oncology, School of Medicine, Southeast University, Nanjing 210009, P.R. China

${ }^{3}$ State Key Laboratory of Natural Medicines, Jiangsu Key Laboratory of Carcinogenesis and Intervention, China Pharmaceutical University, 24 Tongjiaxiang, Nanjing 210009, P.R. China

\begin{abstract}
Many plant-derived natural components have been recognized as effective in the treatment of human diseases. Baicalein (5,6,7-trihydroxyflavone) is one of the primary active components of Scutellaria species, and is well known ingredient in traditional Chinese medicine (TCM) and oriental medicine. Numerous studies have reported that baicalein possesses multiple pharmacological functions, such as anticancer, antioxidant, anti-inflammatory, neuroprotective, hepatoprotective, and antimicrobial properties. The cardioprotective effect of baicalein has been proved in several cell culture and small animal models. This review addresses the recent findings of cardioprotective potentials of baicalein in vitro and in vivo.
\end{abstract}

Keywords: Scutellaria baicalensis georgi; Baicalein; Cardioprotective; Hypertension; Anti-fibrotic; Myocardial ischemia

\section{Introduction}

Life-threatening disorders like coronary heart disease, cerebrovascular, peripheral arterial, rheumatic heart, congenital heart diseases, deep-vein thrombosis, and pulmonary embolism are caused due to impairment of heart and blood vessels, and they are collectively known as cardiovascular diseases (CVDs). The World Health Organization (WHO) has reported that CVDs are the primary cause of mortality worldwide, with an estimated 17.3 million people death in 2008 [1]. According to the American Heart Association (AHA), CVD is the leading cause of deaths in the United States (>800 000 deaths/year) with an estimated annual total cost of $\$ 444$ billion for the treatment [2]. Patients with cardiovascular disease are treated by various medications to reduce hypertension, palpitation, cholesterol, and blood vessel narrowing drugs.

Though these drugs help to combat the severity of the diseases, they trigger serious side effects such as fatigue, shortness of breath, headache, and dizziness [3]. Valsartan is an angiotensin receptor blocker, and used to treat hypertension and heart failure. However it causes unwanted side effects, and may induce myocardial infarction [4]. Statins are the most effective drugs in the treatment of lowering low-density lipoproteins and coronary heart disease. Even though, they do cause cardiomyopathy or rhabdomyolysis and polyneuropathy with adverse side-effects [5]. Hence, there is an urgent need for research and development to design new therapeutic agents with low side-effects for the treatment of cardiovascular diseases.

There has been considerable interest in the role of natural products and their bioactive components in the prevention and treatment of cardiovascular disorders [6]. Recently, many researchers have paid a special attention on the cardioprotective properties of polyphenols. The natural polyphenols exert vasodilator effect and are able to manage lipid profiles in the human system [7].

Scutellaria baicalensis Georgi (Common name: Baikal skullcap and Huang Qin in China), also known as Radix Scutellariae, belongs to the family Lamiaceae. It has been widely used in traditional Chinese medicine (TCM) to treat bacterial, viral infections and cardiovascular diseases [8,9]. Huang Qin-Tang decoction has been used for the treatment of ulcerative colitis, fever, hepatitis, allergic diseases, and hypertension for thousands of years. S. baicalensis and Paeonia lactiflora Pall. (Paeoniaceae) are the key ingredients of this herbal preparation, and comprising of baicalein, wogonoside, baicalin, and wogonin as the active bioflavonoids [10]. Baicalein possesses broad spectrum of biological activity, and a major constituent of the East Asian herbal remedy, "Sho-saiko-to" [11]. In Japan this herbal mixture is popularly used for the treatment of liver diseases [12]. Baicalein also present in Oroxylum indicum (L.) Kurz (Bignoniaceae), which is commonly used in Indian Ayurvedic system of medicine to treat various disorders [13].

It has been reported that baicalein acts as an $\alpha$-glucosidase inhibitor [14], and possesses the following therapeutic properties; anticancer $[15,16]$, osteosarcoma treatment [17], apoptosis induction in human myeloma cells [18], neuroprotective [19], and protection from metabolic syndrome [20]. This review highlights the recent studies which establish the cardioprotective features of baicalein and examines the underlying mechanisms involved in this property.

\section{Baicalein in Cardiovascular System}

Nitric oxide (NO) is an important signaling molecule and free radical, involved in variety of pathophysiological functions. It plays a key role in myocardial ischemia and in myocardial preconditioning [21]. Chen et al. [22] demonstrated that baicalein significantly inhibit the NO production and $i N O S$ gene expression. Mouse macrophage cell line RAW 264.7 was induced by 24 hours incubation of $100 \mathrm{ng} /$

*Corresponding author: Chen Bao-An, Department of Oncology, School of Medicine, Southeast University, Nanjing 210009, P.R. China, Tel: 86-2583272006; Fax+86-25-83272011; E-mail: cba8888@hotmail.com

Received December 04, 2013; Accepted January 03, 2014; Published January 09, 2014

Citation: Bao-An C, Senthilkumar R, Rong F, Guo QL (2014) Cardioprotective Potential of Baicalein: A Short Review of In Vitro and In Vivo Studies. Pharm Anal Acta 5: 280. doi: 10.4172/2153-2435.1000280

Copyright: (c) 2014 Bao-An C, et al. This is an open-access article distributed under the terms of the Creative Commons Attribution License, which permits unrestricted use, distribution, and reproduction in any medium, provided the original author and source are credited. 
$\mathrm{mL}$ lipopolysaccharides (LPS). The cells were treated with different concentrations of baicalein $(20$ and $40 \mu \mathrm{M})$ and investigated the nitrite accumulation in the culture medium. As a result baicalein showed concentration dependent inhibition of nitrite production $\left(\mathrm{IC}_{50} 19.4 \pm 1.0 \mu \mathrm{M}\right)$. At the same time baicalein did not affect the viability of macrophages. Western blot analysis obviously revealed that NO production and inducible nitric oxide synthase (iNOS) gene expression was inhibited by baicalein in concentration dependent manner. However, this compound did not affect iNOS enzyme activity. Furthermore, co-treatment with NOS inhibitors such as $\mathrm{N}$-nitroL-arginine (NLA) and N-nitro-L-arginine methyl ester (L-NAME) enhanced LPS-induced $i N O S$ gene expression, which was also inhibited by baicalein.

\section{San-Huang-Xie-Xin-Tang}

San-Huang-Xie-Xin-Tang (SHXT) is a Chinese herbal remedy comprising Scutellariae radix as a main constituent. This was examined for their cardioprotective effects in both animal model (Wistar rats) and cell lines (RAW 264.7). The LPS stimulated arterial hypotension was inhibited by 0.01 and $0.03 \mathrm{~g} / \mathrm{kg}$ of SHXT as it was given before the administration of LPS. Baicalein is one of the active ingredients of this formula and prevented LPS-induced arterial hypotension, mediated by the inhibition of iNOS and COX-2 gene expression [23]

Liou et al. [24] demonstrated the effect of SHXT in a rat model of acute myocardial apoptosis induced by ischemia/reperfusion (I/R). Both intravenous and oral SHXT treatment reduced the infarct size dose-dependently when compared with the vehicle group. SHXT also decreased the apoptosis with increased Bcl-2/ Bax (an apoptosis inhibitor/an apoptosis promotor) ratio. The SHXT counteracted the I/R-induced downstream signaling, as the consequence, increased myocardial eNOS expression and decreased activation of ERK1/2, p38 and JNK pathways. Baicalein might be a potential active compound present in SHXT and responsible to prevent arterial hypotension and rat hearts from ischemia.

\section{Pro-oxidant Effect of Baicalein}

In general, flavonoids are known to exhibit free radical scavenging activity, and attenuate oxidative stress in cells. But Woo et al. [25] demonstrated an alternative protective mechanism of baicalein in cardiomyocytes. The effects of baicalein and antioxdative enzymes during hypoxia were analyzed by hypoxia/reoxygenation $(H / R)$ treatment. Cardiomyocetes isolated from newborn Sprague Dawley (SD) rats were subjected to hypoxia by providing $0.5 \%$ of oxygen for 8 hours. After that the cells were incubated under normaxia for an hour. Pretreatment with $10 \mu \mathrm{M}$ baicalein reduced lactate dehydrogenase (LDH), and also increased the survival rate of cardiomyocetes during $\mathrm{H} / \mathrm{R}$. N-acetylcysteine (NAC) treatment with baicalein attenuated the protective effect, and concomitantly increased intracellular reactive oxygen species (ROS) level. It implies that baicalein was autoxidized with concomitant generation of hydrogen peroxide, and in addition NAC could amplify this effect. Studies on antioxidant enzymes such as superoxide dismutase (SOD), glutathione (GSH), and catalase (CAT) revealed the involvement of hydrogen peroxide in the cardioprotective mechanism. This experiment demonstrates that the importance of the prooxidant effect of baicalein in cardioprotection.

\section{Cardiovascular Deficits Lead to Cerebral Hypoperfusion}

Heart failure is associated with impaired cognitive function characterized, mainly as delirium and mild cognitive impairment [26]. CVD has become an important risk factor in the development of cognitive decline, and Alzheimer's disease. Cerebral hypoperfusion is induced by cardiovascular deficits including atrial fibrillation, thrombotic events, hypertension, low cardiac index, and valvular pathology [27]. Liu et al. [28] observed that baicalein improves cognitive impairment, and neuronal degeneration in a rat model which mimics cerebrovascular hypoperfusion called 2 VO (permanent occlusion of bilateral common carotid arteries). Intraperitonial administration of 2 to $4 \mathrm{mg} / \mathrm{kg}$ baicalein to the animals daily notably ameliorates the cognitive deficits and neuropathological changes. Long term treatments with baicalein attenuated the free radical formation, and compensate the antioxidant system. This study shows that neuroprotective functions of baicalein might be governed by its antioxidant action.

\section{Anti-fibrotic Effect}

Fibrosis is the formation of excess fibrous connective tissue in an organ or tissue in a reparative or reactive process. Myocardial interstitial fibrosis is a structural determinant of left ventricular (LV) stiffness and diastolic dysfunction. Administration of baicalein $(200 \mathrm{mg} / \mathrm{kg} /$ day $)$ to spontaneously hypertensive rats and normotensive Wistar-Kyoto rats for 12 weeks significantly reduced the LV procollagen expression. Collagen volume fraction of baicalein fed rats $(-2.00 \pm 0.44 \%)$ was comparable to standard drug valsartan treated $(-1.56 \pm 0.46 \%)$ group. Further, the echocardiography results revealed that systolic and diastolic intraventricular septum thickness was also significantly reduced $[29,30]$. This finding suggested that long term administration of baicalein exerts antifibrotic effect in animal model.

\section{Baicalein Protects Chicken Embryonic Cardiomyocytes}

Embryonic ventricular cardiomycetes were prepared from 11day old chick embryo. The cardioprotective effect of baicalein was carried out in ischemia-reperfusion model. Treatment with baicalein $(0.1$ to $5 \mu \mathrm{M})$ was reduced hypoxia-reoxygenationinduced myocardial death in concentration dependent manner and attenuated the apoptosis. Pretreatment with opioid receptor antagonist (naloxone), opioid $\mu$-receptor ( $\beta$-funaltrexamine) and $\delta$-receptor (7-Benzylidenenaltrexone), and protein kinase $\mathrm{C}$ blocked the baicalein's beneficial effect. Gene expression analysis exhibited that the presence of opioid receptors mRNA in chicken embryonic cardiomyocytes, and protective activity was mediated by $\mu$ and $\delta$ receptor related signaling pathways [31].

\section{Against Doxorubicin-Induced Cardiotoxicity}

Doxorubicin (DXR) or a driamycin is an anticancer anthracycline antibiotic that presents a dose-dependent and additive cardiotoxicity as serious side effect [32]. Adriamycin administration in rats causes cardiomyopathy and congestive heart failure [33]. Cardiotoxic sideeffects represent a serious complication of anticancer therapy with doxorubicin, and various mechanisms have been proposed to explain DXR-induced cardiotoxicity [34]. DXR $(10 \mu \mathrm{M})$ increased cell death, DNA fragmentation and the phosphorylation of ROS-sensitive proapoptotic kinase c-Jun N-terminal kinase (JNK) in chick cardiomyocyte model. Adjunct treatment of baicalein $(25 \mu \mathrm{M})$ and doxorubicin significantly reduced both free radical generation and cell death by inhibiting apoptosis. It also weakened the dissipated mitochondrial potential and increased DNA fragmentation. In addition, concurrent baicalein treatment did not affect the anti-proliferative effect of doxorubicin in human breast cancer MCF-7 cells [35]. This study demonstrated that baicalein protects cardiomyocetes from DXR toxicity by quenching radicals and preventing apoptosis. 


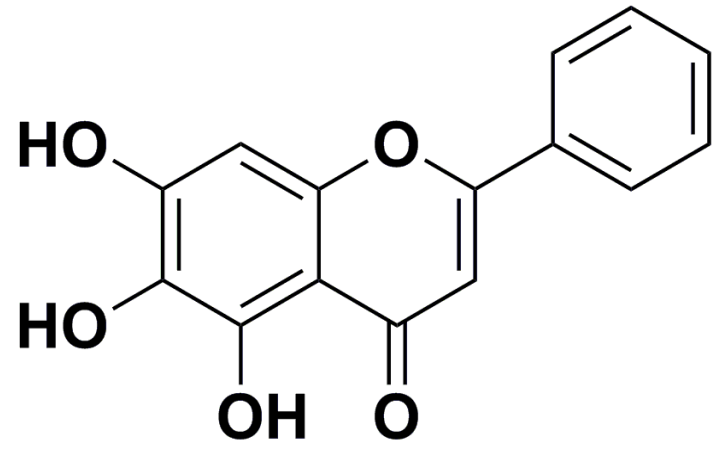

Figure 1: Chemical structure of Baicalein.

\section{Cardiac Contractile Function}

The interest in the study of endotoxemia in the clinical science was attracted as a result of incidence of sepsis in animal models [36]. Myocardial depression is a well known indication of organ dysfunction in sepsis. Baicalein $(10 \mathrm{mg} / \mathrm{kg})$ was administered intravenously to Wistar rats $30 \mathrm{~min}$ after induced with $10 \mathrm{mg} / \mathrm{kg}$ LPS (Figure 1). The contractile function of the isolated heart was examined, and cardiac protein expression related to inflammatory responses, superoxide anion production and caspase-3 were also measured. Baicalein significantly attenuated the LPS-induced hypotension through heme oxygenase- 1 induction and reduced superoxide anion formation in the myocardium of rats [37]. This study noticeably indicates that baicalein improves cardiac contractility in LPS-induced sepsis by the suppression of inflammatory markers, and inhibition of apoptosis.

\section{Protects Against Endothelin-1}

Endothelin-1 (ET-1) is a peptide hormone, vasoconstrictor secreted by endothelial cells, which acts as the natural counterpart of the vasodilator NO [38,39]. It has associated with human aging, including hypertension, Type 2 diabetes and coronary artery disease [40]. Lin et al. [41] studied the antiproliferative effects of baicalein on ET-1 mediated pulmonary artery smooth muscle cells (PASMCs) which would contribute to pulmonary arterial hypertension (PAH).

Intrapulmonary artery smooth muscle cells were isolated and cultured from female SD rats. Baicalein $(0.3$ to $3 \mu \mathrm{M})$ inhibited PASMCs proliferation, promoted cell cycle progression, enhanced $\left[\mathrm{Ca}^{2+}\right]$ i levels, upregulated the canonical transient receptor potential 1 (TRPC1) channel and membrane protein kinase $\mathrm{C}$ expression induced by ET-1 [41]. This observation suggests that baicalein exhibited antiproliferative activity on ET-1 induced PASMC, and could be potential agent to PAH.

\section{Neonatal Cardiomyocyte Injury}

Jun et al. [42] observed the effect of baicalein on myocardial ischemia in male $\mathrm{SD}$ rats caused by coronary artery ligation, and $\mathrm{Na}_{2} \mathrm{~S}_{2} \mathrm{O}_{4}$-induced $\mathrm{H} / \mathrm{R}$ injury of neonaltal cardiomyocytes. Animals were pretreated with intragastric administration of different concentrations baicalein $(40,80$ and $160 \mathrm{mg} / \mathrm{kg})$ for 7 days. Pretreatment with baicalein $(160 \mathrm{mg} / \mathrm{kg})$ effectively inhibited ischemia induced elevation of $J$ point in ECG, and diminished $\mathrm{CK}, \mathrm{LDH}$ and MDA in serum, and also decreased serum SOD activity in a dose-dependent fashion. Baicalein pretreatment in vitro increased the viability of $\mathrm{H}_{2} \mathrm{O}_{2}$-injured cardiomyocytes, and decreased the leakage of $\mathrm{LDH}$. Hence, pretreatment with baicalein has the ability to protect rat myocardium from ischemia and neonatal cardiomyocetes.

\section{Mitochondrial Damage Mediated Apoptosis}

Wang et al. [43] observed the effect of baicalin pretreatment on reperfusion injury in vivo, and possible underlying mechamisms. Coronary artery occlusion and reperfusion in mice were performed. Baicalein $(100 \mathrm{mg} / \mathrm{kg})$ was administered via the left jugular vein 10 min before left anterior descending coronary artery (LAD) ligation. After 2 hours of reperfusion, area at risk (AAR) and infarct size (INF) were studied by heart staining of Evans blue and tetrazolium chloride to determine. In addition, apoptosis was determined by TUNEL assay. The results of this study showed that the ratios of INF to AAR $(46.75 \pm 1.94 \%)$ were significantly decreased in the baicalin group. In addition, baicalein dramatically reduced the mitochondrial damage, and associated with mitochondria mediated apoptosis.

\section{Adverse effects of Baicalein}

Endothelium-derived relaxing factor (EDRF) is an unstable substance produced from artery and vein that mediates the functions of endothelium-dependent vasodilators. But $\mathrm{NO}$ is an unstable endothelium-independent vasodilator that is released from vasodilator drugs such as nitroprusside and glyceryl trinitrate. It was identified by experiments that EDRF behaves like NO and labile nitroso species [44] NO and cyclic guanosine monophosphate (GMP) consist of many key roles in physiological regulation, including smooth muscle relaxation, visual transduction, intestinal ion transport, platelet function and biochemical interactions [45]. In contrast, acute exposure or lower concentration of baicalein enhances sensitivity to receptor dependent vasoconstrictor agonists in rat arteries [46].

Huang et al. [47] observed the effect of baicalein on endotheliumdependent and -independent relaxation in SD rat excised aortic rings. At the end of experiment they found baicalein impaired the endothelium independent relaxation by NO donors. Baicalein at $50 \mu \mathrm{M}$ significantly reduced both acetylcholine and cyclopiazonic acid induced relaxation. Baicalein also reduced the cyclic GMP levels stimulated by acetylcholine in endothelium-intact rings. Acute exposure to baicalein $(10 \mu \mathrm{M})$ impairs eNOS/endothelium-derived nitric oxide-mediated vascular tone in isolated aortic rings from normal Wistar-Kyoto rats through the inhibition of endothelium-derived nitric oxide and cyclooxygenasemediated release of superoxide anions [48].

\section{Conclusion}

Drug development and clinical use of plant derived compounds have improved dramatically in recent years. In this review, the main molecular mechanisms underlying the cardioprotective properties of baicalein have been concisely reported. In vitro and in vivo studies demonstrate that baicalein, a naturally occurring flavonoid which improves the cognitive deficits, neuropathological changes, antioxitant activity, and acts as antifibrotic agent. It protects cardiomyocetes via many signal transduction pathways. Further clinical studies are greatly required for the development of baicalein as promising drug to treat cardiovascular diseases.

\section{References}

1. Global status report on non-communicable diseases (2010) World Health Organization, Geneva, 2011.

2. Roger VL, Go AS, Lloyd-Jones DM, Benjamin EJ, Berry JD, et al. (2012) Heart disease and stroke statistics-2012 update: a report from the American Heart Association. Circulation 125: e2-e220

3. Toyoshima H, Takahashi K, Akera T (1997) The impact of side effects on hypertension management: a Japanese survey. Clin Ther 19: 1458-1469. 
Citation: Bao-An C, Senthilkumar R, Rong F, Guo QL (2014) Cardioprotective Potential of Baicalein: A Short Review of In Vitro and In Vivo Studies. Pharm Anal Acta 5: 280. doi: 10.4172/2153-2435.1000280

4. Verma S, Strauss M (2004) Angiotensin receptor blockers and myocardial infarction. BMJ 329: 1248-1249.

5. Moosmann B, Behl C (2004) Selenoprotein synthesis and side-effects of statins. Lancet 363: 892-894.

6. Vilahur G, Badimon L (2013) Antiplatelet properties of natural products. Vasc Pharmacol 59: 67-75.

7. Quinones M, Miguel M, Aleixandre A (2013) Beneficial effects of polyphenols on cardiovascular disease. Pharmacol Res 68: 125-131.

8. Huang Y, Tsang SY, Yao X, Chen ZY (2005) Biological properties of baicalein in cardiovascular system. Curr Drug Targets Cardiovasc Haematol Disord 5 $177-184$

9. Li-Weber M (2009) New therapeutic aspects of flavones: the anticancer properties of Scutellaria and its main active constituents Wogonin, Baicalein and Baicalin. Cancer Treat Rev 35: 57-68.

10. Zhang ZQ, Liua W, Zhuang L, Wang J, Zhang S (2013) Comparative pharmacokinetics of Baicalin, Wogonoside, Baicalein and Wogonin in plasma after oral administration of pure Baicalin, Radix Scutellariae and ScutellariaePaeoniae couple extracts in normal and ulcerative colitis rats. Iran J Pharm Res 12: 399-409.

11. Rossi M, Meyer R, Constantinou P, Caruso F, Castelbuono D, et al. (2001) Molecular structure and activity toward DNA of baicalein, a flavone constituent of the Asian herbal medicine "Sho-saiko-to". J Nat Prod 64: 26-31.

12. Okita K, Li Q, Murakamio T, Takahashi M (1993) Anti-growth effects with components of sho-saiko-to (TJ-9) on cultured human hepatoma cells. Eur J Cancer Prev 2: 169-175.

13. Rahul Dev L, Anurag M, Rajiv G (2010) Oroxylum indicum: A Review. Phcog J 2: $304-310$

14. Nishioka T, Kawabata J, Aoyama Y (1998) Baicalein, an a-glucosidase inhibitor from Scutellaria baicalensis. J Nat Prod 61: 1413-1415.

15. Liu C, Wu J, Xu K, Cai F, Gu J, et al. (2010) Neuroprotection by baicalein in ischemic brain injury involves PTEN/AKT pathway. J Neurochem 112: 1500 1512.

16. Huang WS, Kuo YH, Chin CC, Wang JY, Yu HR, et al. (2012) Proteomic analysis of the effects of baicalein on colorectal cancer cells. Proteomics 12: 810-819.

17. Zhang Y, Song L, Cai L, Wei R, Hu H, et al. (2013) Effects of baicalein on apoptosis, cell cycle arrest, migration and invasion of osteosarcoma cells. Food Chem Toxicol 53: 325-333.

18. Ma Z, Otsuyama K, Liu S, Abroun S, Ishikawa H, et al. (2005) Baicalein, a component of Scutellaria radix from Huang-Lian-Jie-Du-Tang (HLJDT), leads to suppression of proliferation and induction of apoptosis in human myeloma cells. Blood 105: 3312-3318.

19. Leyen KV, Kim HY, Lee SR, Jin G, Arai K, et al. (2006) Baicalein and 12/15-lipoxygenase in the ischemic brain. Stroke 37: 3014-3018.

20. Pu P, Wang XA, Salim M, Zhu LH, Wang L, et al. (2012) Baicalein, a natura product, selectively activating $\mathrm{AMPKa}_{2}$ and ameliorates metabolic disorder in diet-induced mice. Mol Cell Endocrinol 362: 128-138.

21. Bolli R (2001) Cardioprotective function of inducible Nitric oxide synthase and role of nitric oxide in myocardial ischemia and preconditioning: an overview of a decade of research. J Mol Cell Cardiol 33: 1897-1918.

22. Chen YC, Shen SC, Chen LG, Lee TJF, Yang LL (2001) Wogonin, baicalin, and baicalein inhibition of inducible nitric oxide synthase and cyclooxygenase-2 gene expressions induced by nitric oxide synthase inhibitors and lipopolysaccharide. Biochem Pharmacol 61: 1417-1427.

23. Lo YC, Tsai PL, Huang YB, Shen KP, Tsai YH, et al. (2005) San-Huang-XieXin-Tang reduces lipopolysaccharides-induced hypotension and inflammatory mediators. J Ethnopharmacol 96: 99-106.

24. Liou SF, Ke HJ, Hsu JH, Liang JC, Lin HH, et al. (2011) San-Huang-Xie-XinTang prevents rat hearts from ischemia/reperfusion-induced apoptosis through eNOS and MAPK pathways. Evid Based Complement Alternat Med 915051 $1-9$.

25. Woo AY, Cheng CH, Waye MM (2005) Baicalein protects rat cardiomyocytes from hypoxia/reoxygenation damage via a prooxidant mechanism. Cardiovasc Res 65: 244- 253.

26. Heckman GA, Patterson CJ, Demers C, St.Onge J, Turpie ID, et al. (2007) Heart failure and cognitive impairment: Challenges and opportunities. Clin Interv Aging 2: 209-218.
27. De la Torre JC (2012) Cardiovascular risk factors promote brain hypoperfusion leading to cognitive decline and dementia. Cardiovasc Psychiatry Neuro 367516.

28. Liu C, Wu J, Gu J, Xiong Z, Wang F, et al. (2007) Baicalein improves cognitive deficits induced by chronic cerebral hypoperfusion in rats. Pharmacol Biochem Behav 86: 423-430.

29. Kong EKC, Huang Y, Sanderson JE, Chen KB, Yu CM (2008) Anti-fibrotic effect of Baicalein beyond blood pressure control in a rat model of genetic hypertension. Int J Cardiol 125: S39-S50.

30. Kong EK, Yu S, Sanderson JE, Chen KB, Huang Y, et al. (2011) A novel antifibrotic agent, baicalein, for the treatment of myocardial fibrosis in spontaneously hypertensive rats. Eur J Pharmacol 658: 175-181.

31. Tu IH, Yen HT, Cheng HW, Chiu JH (2008) Baicalein protects chicken embryonic cardiomyocyte against hypoxia-reoxygenation injury via $\mu$ - and $\delta$ but not K-opioid receptor signaling. Eur J Pharmacol 588: 251-258.

32. Carvalho FS, Burgeiro A, Garcia R, Moreno AJ, Carvalho RA, et al. (2013) Doxorubicin-induced cardiotoxicity: From bioenergetic failure and cell death to cardiomyopathy. Med Res Rev 34: 106-135.

33. Mettler FP, Young DM, Ward JM (1977) Adriamycin induced cardiotoxicity (Cardiomyopathy and congestive heart failure) in rats. Cancer Res 37: 2705 2713.

34. Tokarska-Schlattner M, Zaugg M, Zuppinger C, Wallimann T, Schlattner U (2006) New insights into doxorubicin-induced cardiotoxicity: The critical role of cellular energetic. J Mol Cell Cardiol 41: 389-405.

35. Chang WT, Li J, Huang HH, Liu H, Han M, et al. (2011) Baicalein protects against doxorubicin-induced cardiotoxicity by attenuation of mitochondrial oxidant injury and JNK activation. J Cell Biochem 112: 2873-2881.

36. Redl H, Bahrami S, Schlag G, Traber DL (1993) Clinical detection of LPS and animal models of endotoxemia. Immunobiology 187: 330-345.

37. Lee YM, Cheng PY, Chim LS, Kung CW, Ka SM, et al. (2011) Baicalein an active component of Scutellaria baicalensis Georgi, improves cardiac contractile function in endotoxaemic rats via induction of heme oxygenase- 1 and suppression of inflammatory responses. J Ethnopharmacol 135: 179-185.

38. Marasciulo FL, Montagnani M, Potenza MA (2006) Endothelin-1: the yin and yang on vascular function. Curr Med Chem 13: 1655-1665

39. Stow LR, Jacobs ME, Wingo CS, Cain BD (2011) Endothelin-1 gene regulation. FASEB J. 25: 16-28

40. Westby CM, Weil BR, Greiner JJ, Stauffer BL, DeSouza CA (2011) Endothelin-1 vasoconstriction and the age-related decline in endothelium-dependent vasodilatation in men. Clin Sci $120: 485-491$.

41. Lin YL, Lin RJ, Shen KP, Dai ZK, Chen IJ, et al. (2011) Baicalein, isolated from Scutellaria baicalensis, protects against endothelin-1-induced pulmonary artery smooth muscle cell proliferation via inhibition of TRPC1 channel expression. $J$ Ethnopharmacol 138: 373-381.

42. Jun P, Wen-Jie X, Bi-Xia H, Yan L, Hong Z, et al. (2011) Effects of Baicalein on rat myocardial ischemia and neonatal cardiomyocyte injury. Chin J Nat Med 9: $132-140$.

43. Wang X, He F, Liao Y, Song X, Zhang M, et al. (2013) Baicalin pretreatment protects against myocardial ischemia/reperfusion injury by inhibiting mitochondrial damage-mediated apoptosis. Int J Cardiol 168: 4343-4345.

44. Ignarro LJ, Buga GM, Wood KS, Byrns RE, Chaudhuri G (1987) Endotheliumderived relaxing factor produced and released from artery and vein is nitric oxide. Proc Natl Acad Sci 84: 9265-9269.

45. McDonald LJ, Murad F (1996) Nitric oxide and cyclic GMP signaling. Proc Soc Exp Biol Med 211: 1-6.

46. Chen ZY, Su YL, Lau CW, Law WI, Huang Y (1999) Endothelium-dependent contraction and direct relaxation induced by baicalein in rat mesenteric artery. Eur J Pharmacol 374: 41-47.

47. Huang Y, Wong CM, Lau CW, Yao X, Tsang SY, et al. (2004) Inhibition of nitric oxide/cyclic GMP-mediated relaxation by purified flavonoids, baicalin and baicalein, in rat aortic rings. Biochem Pharmacol 67: 787-794.

48. Machha A, Achike FI, Mohd MA, Mustafa MR (2007) Baicalein impairs vascula tone in normal rat aortas: Role of superoxide anions. Eur J Pharmacol 565: 144-150. 